\title{
A!
}

This is an electronic reprint of the original article.

This reprint may differ from the original in pagination and typographic detail.

Volynets, Sofia; Glerean, Enrico; Hietanen, Jari K; Hari, Riitta; Nummenmaa, Lauri

\section{Bodily maps of emotions are culturally universal}

\section{Published in:}

EMOTION

DOI:

$10.1037 / \mathrm{emo} 0000624$

Published: 01/10/2020

Document Version

Peer reviewed version

Please cite the original version:

Volynets, S., Glerean, E., Hietanen, J. K., Hari, R., \& Nummenmaa, L. (2020). Bodily maps of emotions are culturally universal. EMOTION, 20(7), 1127-1136. https://doi.org/10.1037/emo0000624

This material is protected by copyright and other intellectual property rights, and duplication or sale of all or part of any of the repository collections is not permitted, except that material may be duplicated by you for your research use or educational purposes in electronic or print form. You must obtain permission for any other use. Electronic or print copies may not be offered, whether for sale or otherwise to anyone who is not an authorised user. 


\section{Bodily maps of emotions are culturally universal}

Sofia Volynets ${ }^{1}$, Enrico Glerean ${ }^{1,2}$, Jari K. Hietanen ${ }^{3}$, Riitta Hari ${ }^{4}$, and Lauri Nummenmaa ${ }^{2,5^{*}}$

${ }^{1}$ Department of Neuroscience and Biomedical Engineering; School of Science, Aalto University

${ }^{2}$ Turku PET Centre, University of Turku; Turku; Finland

${ }^{3}$ Faculty of Social Sciences/Psychology, University of Tampere

${ }^{4}$ Department of Art, School of Arts, Design and Architecture, Aalto University, Finland

${ }^{5}$ Department of Psychology; University of Turku; Turku; Finland

Author contributions: Designed experiments: LN, RH, JH, EG. Acquired data: LN and EG. Analysed data: SV, EG wrote manuscript: LN, RH, JH, SV and EG.

\section{*Corresponding author}

Lauri Nummenmaa

Turku PET Centre and Department of Psychology

Kiinamyllynkatu 4-6, FI-20520 Turku, Finland

Email: latanu@utu.fi 


\begin{abstract}
Emotions are often felt in the body, and interoceptive feedback is an important component of conscious emotional experiences. Here, we provide support for cultural universality of bodily sensations associated with 13 emotions in a large international sample (3954 individuals from 101 countries, age range 18-90). Subjects were presented with two silhouettes of bodies alongside with emotional words and asked to color the bodily regions whose activity they felt increasing or decreasing while they experienced each given emotion. We tested the effects of various background factors (age, sex, education, body-mass index, nationality, civilization, and language) on the bodily sensation maps. Bodily sensations associated with emotions were concordant across the tested cultures $\left(r_{s}>0.82\right)$ and across the sexes $(r>0.80)$. Bodily sensations weakened during ageing (mean $r_{s}=0.11$ across emotions). We conclude that universality in experiencing emotions in the body is stronger than the differences due to culture or sex.
\end{abstract}

Keywords: Emotion, somatosensation, body, culture, universalism, ageing 


\section{Introduction}

Emotions prepare us for action. The mechanisms of such survival responses rely on numerous synchronized changes in cardiovascular, skeletomuscular, gastrointestinal, respiratory, endo- and exocrine, and autonomic nervous systems (ANS; Kreibig, 2010; Levenson, 2003) as well as on distributed neural circuitries in the brain (Kragel et al., 2016; Saarimaki et al., 2018; Saarimäki et al., 2018; Saarimäki et al., 2016). Interoceptive and somatosensory processes in turn play a major role in the generation of subjectively felt emotion states (Damasio et al., 2000; Kreibig, 2010; Nummenmaa et al., 2014). Emotions are physiological survival functions, only modestly influenced by cultural factors and individual differences in geno- and phenotype (Ekman, 1992; Sauter et al., 2010).

Emotions are inherently social and adaptive, and henceforth not completely devoid of cultural influence and learning-dependent plasticity. Accordingly, cultural variation is observed in social dimensions of emotions such as facial expression recognition (Jack et al., 2012; Nelson \& Russell, 2013), display rules (Matsumoto et al., 2008) and subjective feelings of social emotions (Kitayama et al., 2006). There exist also significant differences in how different individuals, in particular men and women, within one culture process emotions (Fischer et al., 2004). In general, women recognize emotional expressions better and express emotions more than men do, whereas men respond more strongly to threatening cues (Kret \& De Gelder, 2012). Similarly, vocal and facial emotion-recognition ability (Mill et al., 2009) as well as emotional expressivity decline during ageing, potentially explaining the general impairments in recognizing older peoples' emotional expressions (Folster et al., 2014).

Recently it has been shown that bodily "feeling fingerprints" determine the qualia of subjective emotional feelings (Nummenmaa et al., 2014; Nummenmaa et al., 2018; Torregrossa et al., 2018). Yet, cultural universalism, sex differences, and age dependency these bodily feelings of emotions remains poorly understood, and the existence of emotion-specific, universal psychophysiological signatures of emotions is currently debated (Kreibig, 2010; Siegel et al., 2018). 
Unlike facial expression recognition tasks or psychophysiological measurements, high-resolution topographical mapping of bodily sensations provides a straightforward measure of one potentially universal component of human emotions. Subjective emotional feelings and their expressions are only modestly correlated with the underlying autonomic activity (Anderson et al., 2018; Mauss et al., 2005), potentially due to the fact that these studies typically measure general-level autonomic activity rather than, for example, multichannel cardiovascular, respiratory and endo- and exocrine function as well as bodily region specific skeletomuscular activity. Focussing on specifically at the output layer of these functions - the subjectively felt bodily changes - circumvents this problem even though it sacrifices the physiological specificity.

Furthermore, many facial expressions may be used primarily for affiliative purposes rather than for genuinely communicating one's emotional state (Manninen et al., 2017; Scott et al., 2015). They are also oftentimes visible to others, providing ample opportunities for learning cultural and sex-specific display rules. In turn, physiological manifestations of emotions difficult to recognize from others by simple observation: Even though some internal states can be inferred directly from behaviour (e.g. an erection indexes sexual arousal and shivering lowered body temperature), internal states are in general less visible than emotional expressions that are meant to be seen by others. Thus, even though experience-dependent plasticity might lead to culturally distinct expressions of emotions via action-observation based learning, bodily states and the corresponding subjective bodily sensations associated with distinct emotions could be less likely to be influenced by culture.

We have previously tested concordance of embodied emotions across one West European (Finland) and one East Asian (Taiwan) countries (Nummenmaa et al., 2014). Despite concordant findings across these culturally distant samples, this earlier study did not sample the cultural variation of bodily felt emotions across the world. Finally, developmental studies have shown that understanding of emotions (Thompson \& Lagattuta, 2006), emotion recognition skills (Widen, 2013) as well the discrete bodily fingerprints of emotions (Hietanen et al., 2015) are tuned to adult- 
like operation during the childhood. Yet, there exists no data on how the bodily feelings of emotions develop through the adult lifespan.

Here we tested the i) cultural universality, ii) sex differences and iii) age-dependence of bodily sensation maps (BSMs) associated with different emotions; the study was based on unique computer-based, topographical self-report method that we have previously validated (emBODY; Nummenmaa et al., 2014; Nummenmaa et al., 2016). To provide strong evidence for the cultural universalism of bodily sensations associated with emotions, we employed a cross-cultural design aiming at maximal geographic and cultural diversity of tested cultures, as statements about universality are strongest when the findings apply to an approximation of the world's database of cultures (Norenzayan \& Heine, 2005).

\section{Materials and Methods}

\section{Subjects}

The study protocol was approved by the Aalto University Institutional Review Board (21022012v8). A total of 6559 individuals (5355 females, 1204 males) from altogether 105 countries registered in the experiment that we advertised on the laboratory webpage, press releases, and social media in English so that subjects could participate from all over the world. The respondents' geographical location (current country of residence) was determined by the IPaddress used in connection to the Aalto University servers. A subset (12.5\%) of the subjects were also directly asked about their nationality, these data showed that IP address and self-reported nationality were, on average, matching on $84 \%$ of cases, likely representative of the whole data. The experiment was presented in English to all subjects regardless of their mother-tongue, because previous work shows that linguistic factors do not confound the responses in this task. Specifically, the bodily maps of emotions are concordant when participants report bodily feelings associated with verbally presented emotion tokens, or when they report their actual bodily feelings resulting from nonverbal emotion induction (Nummenmaa et al., 2014). Upon logging to the experiment and providing informed consent, subjects reported background information including age, sex, 
handedness, education, height and weight (Table S-1). Subjects under 18 years of age and those completing the body map task for less than 10 emotions were removed from the sample. We also filtered subjects reporting anomalous weights and heights based on Anthropometric Reference Data for Children and Adults in United States, 2011-2014. We took the lowest 5\% and the highest 95\% percentiles for values of height and weight for adults from all examined ages and cultural backgrounds as thresholds, and excluded subjects who reported values outside these limits (43.2$130 \mathrm{~kg}$ and $144-176.5 \mathrm{~cm}$ for females, $48.5-137.3 \mathrm{~kg}$ and $155-188.9 \mathrm{~cm}$ for males). We also removed the subjects whose reported nationality mismatched their IP-address-based geographical location while they completed the experiment. Finally, the data were manually screened for abnormal painting behaviour (e.g., drawing symbols or doodling on the bodies). The resulting final sample included data from 3954 individuals from 101 countries aged 18-90 ( $M=34.9$ years; 3260 females, 694 males). To reveal potential cultural factors underlying variation in the emotion-specific BSMs, we divided subjects into 9 groups according to major civilizations (rather than modelling cultural variation as a function of country of residence only), based on their country of residence, as defined in (Huntington, 1996). Due to limited number of subjects in some groups, we also analysed the data by collapsing these groups into Western and Non-Western cultures.

\section{Data acquisition}

Data were acquired online using the emBODY tool (Figure 1) developed by our group and made freely available at https://version.aalto.fi/gitlab/eglerean/embody. In this computerized tool, subjects were presented with two silhouettes of a human body and an emotion term between them. Subjects' task was to read the emotion word and denote in the silhouettes the areas in their body where they would feel that activation was increased (on the left-side body) and decreased (on the right-side body) when experiencing that emotion. Subjects evaluated their bodily sensations associated with six basic (Per Ekman's original definition: anger, fear, disgust, happiness, sadness, and surprise) and seven nonbasic (anxiety, love, depression, contempt, pride, shame, and jealousy) 
emotions as well as the neutral state. Each word was presented during the experiment once on separate trials in random order. Previous work suggests that the results from this type of a brief word-cue based task are concordant with the sensation maps reported during actual experimentally-induced emotions (Nummenmaa et al., 2014). The total area for the bodies was 50,365 pixels, and the diameter of the painting tool was 12 pixels. Painting was dynamic and successive strokes on a region increased the opacity of the paint until the maximum value (arbitrarily set at 255) was reached, shown to participant as RGB colour [255,0,0] for activation and $[0,0,255]$ for deactivation. Finished images were stored in matrices with positive values for painted activations and negative values for deactivations.

\section{Data availability}

Full dataset is available on Zenodo (link updated if manuscript is accepted)

\section{Statistical analysis}

Subject- and emotion-wise activation and deactivation maps for each emotion were first combined into bodily sensation maps (BSMs) representing both activations (positive values) and deactivations (negative values). Responses outside the body area were masked. Mass univariate $t$-tests were then used on the subjectwise BSMs to compare pixelwise activations and deactivations of the BSMs for each emotional state against zero. This procedure resulted in statistical $t$-maps, where pixel intensities reflect statistically significant reported bodily changes associated with each emotional state across all the subjects. False discovery rate (FDR) correction with an alpha level of 0.05 was applied to the statistical maps to control for false positives due to multiple comparisons here and in all following analyses. 


\section{A. Initial screen with blank bodies B. Subject-wise colored \\ For the picture below, evaluate how the activity of your body changes when you feel.. \\ activation and deactivation maps}

C. Subject-wise combined

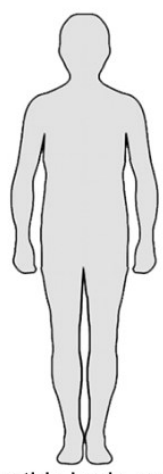

For this body, color you feel increasing or getting stronger Reset
Love

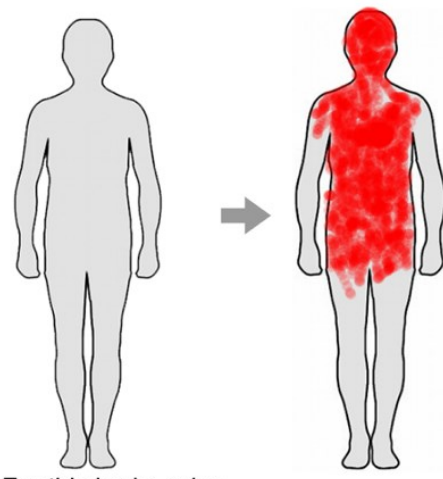

For this body, color the regions whose activity
you feel decreasing or getting weaker Click here when done
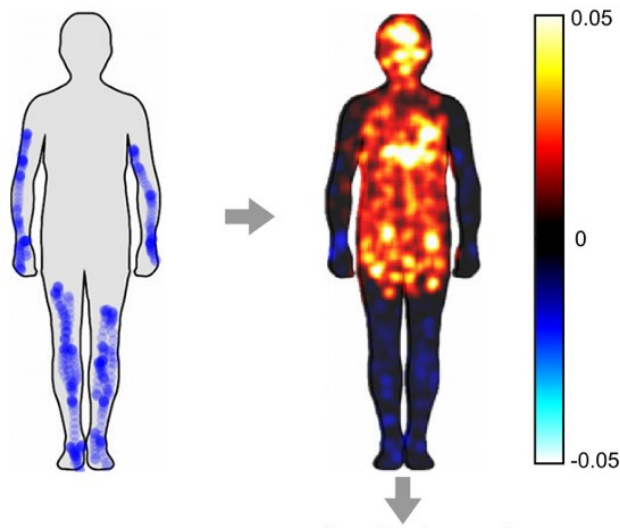

Analysis and statistical inference

Figure 1. Experimental protocol. Subjects were presented with blank body silhouettes (A) and were asked to color the regions whose activity they felt increasing (left body) and decreasing (right body) during a given emotion (B). Activation and deactivation maps were subsequently combined (C) for statistical analysis, the whole body was represented by 50,291 data points and the coloring data were stored as integers.

To test whether the bodily sensations associated with each emotion are similar across different cultures, we selected from our sample 15 countries with at least 45 subjects in each (Table S-1; total $\mathrm{N}=3085)$. First, we computed the average t-maps of each emotion for each country. Because the number of subjects varied across countries, we converted the t-maps into effect-size maps. To visualize the similarity structure of the emotion-specific BSMs across the selected 15 countries, the emotion-wise effect-size maps were clustered using t-distributed stochastic neighbour embedding (t-SNE; https://lvdmaaten.github.io/tsne/). In the resulting multidimensional scaling projection, distance between the elements (here emotions in each country) is related to their similarity. Next, we examined the inter-nation consistency of BSMs by computing a Spearman-correlation-based similarity matrix of the abovementioned effect-size maps (Fig. 4). We compared all the available country + emotion effect-size maps (14 emotions $* 15$ 
countries $=210$ maps, e.g. happiness in Finland, disgust in USA, and so on), and the resulting matrix was sorted by emotions. We then computed the mean similarity score separately for each emotion across all countries to assess the inter-nation consistency of BSMs for the given emotion (e.g. the mean similarity of "pride" maps across all countries). To test the statistical significance of those mean emotion-wise inter-nation similarity scores (reported in Fig. 4), we shuffled the columns and the rows of the initial similarity matrix 5000 times to compute a surrogate distribution of similarity scores for each emotion.

To deal with multicollinearity of the predictors, we employed ridge regression to evaluate the influence of emotion, demographic and anthropometric (age, sex, education, body-mass index BMI) and cultural (country of residence, civilization, and language family) on the BSMs (Fig. S1). This approach allows non-orthogonal regressors while quantifying how much variance emotions themselves explain in the body maps relative to cultural and subject-specific factors by adding a degree of bias to the regression estimates. For each pixel, the subject and emotionwise values were regressed against a model containing the regressors of interest 14 emotions, 15 countries with $\mathrm{N}>$ 45 plus "other" category, 9 major civilizations according to Huntington (Western, Orthodox, Islamic, Buddhist, Hindu, African, Latin American, Sinic, Japanes), 8 language families accordingly to the 1st official language of the country the subject's IP was in plus "other" category, education, age, BMI, and sex). We used Huntington's classification of cultures because the conventions stemming from national ideologies, politics and religion could presumably have long-term and consistent impact on the way emotions are potentially shaped in the culture. We tested a logarithmic lambda parameter in range $10^{-3}-10^{9}$ and chose $\lambda=10^{7}$ as it provided stable beta values across all pixels. To assess statistical significance and control for multiple comparisons, we shuffled the rows of the model matrix and considered the biggest beta value to obtain the max-statistics distribution .

Finally, we applied a two-sample $t$-test to compare BSMs of Western subjects against those of the others (in Western group $\mathrm{N}=3069$, in Non-Western group $\mathrm{N}=885$ ). A similar test was 
performed to compare BSMs between males and females. The relationship between BSMs and subjects' geographical location (longitude and absolute latitude), antrophometric parameters (height, weight, BMI), and age, were assessed by pixelwise Spearman correlation separately for each emotion.

\section{Results}

Figure 1 shows bodily sensation maps associated with the 13 emotions as well as the neutral state reported by the whole sample $(\mathrm{N}=3954)$, with distinct bodily "fingerprints" for each emotion (see Figure S-1 for standard deviation maps). The similarity space for the emotions across different countries derived by t-SNE (Fig. 2) revealed that the 13 examined emotions plus neutral state formed distinctive clusters, with clear concordance across tested countries. For example, BSMs for "Love" are remarkably similar across all countries, but also distinct from both closely related (e.g. "Happiness") and more distant (Depression) emotions. This concordance of BSMs across cultures was corroborated by similarity analyses of the BSMs for the 15 countries with $\mathrm{N}>$ 45. Despite some variability across the countries, the mean similarity of BSMs for each emotion was considerable across tested countries (emotion-wise intercultural similarities indexed by $r s>$ 0.82, ps $<0.001 ;$ Figure 3). 


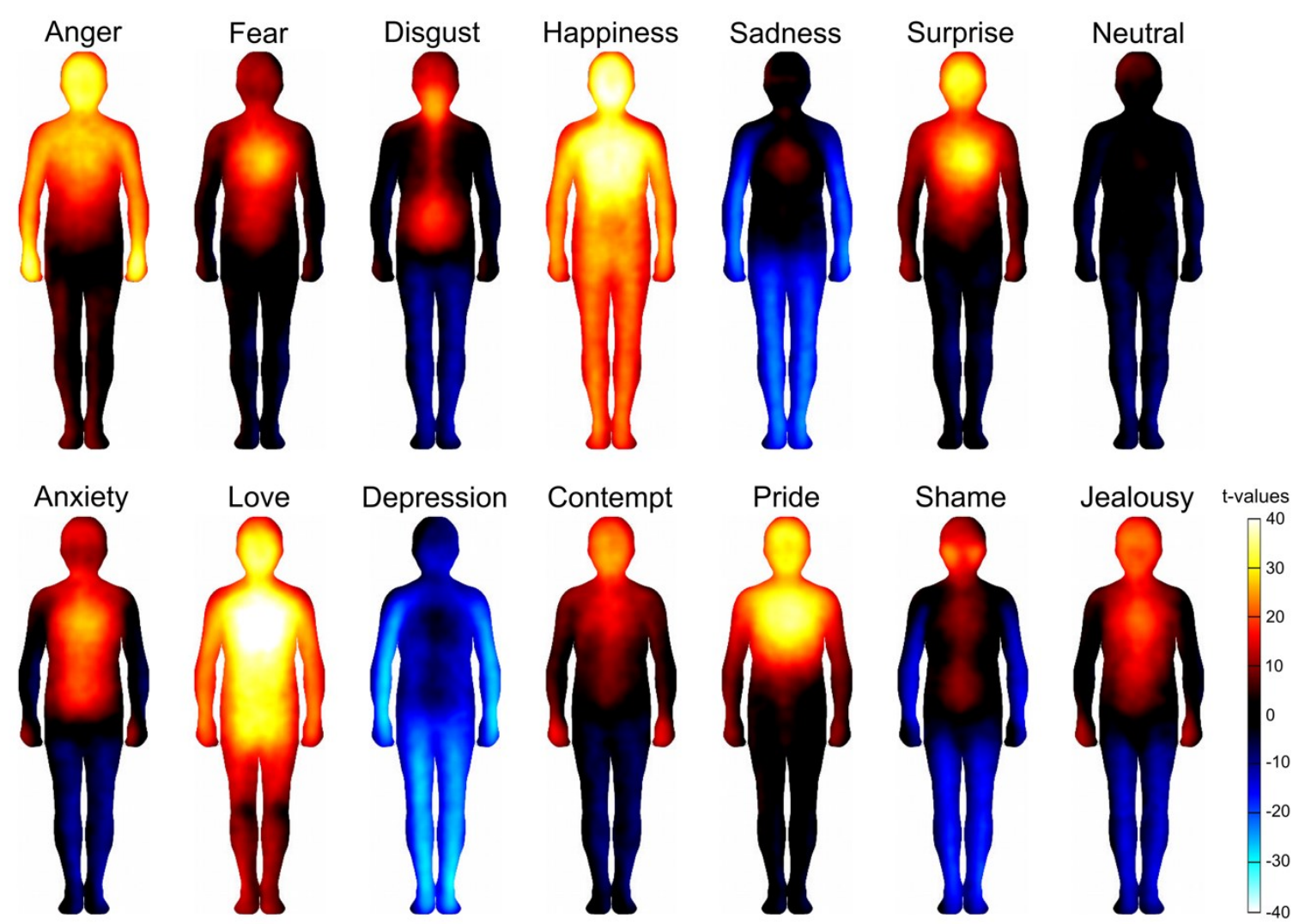

Figure 1. Bodily maps of emotions for the whole sample $(\mathbf{N}=3954)$. The body maps show regions whose activation increased (warm colours) or decreased (cool colors) when the subject was feeling the indicated emotion $(p<0.05$, FDR corrected; $t>2.92)$. The colorbar indicates the $t$-statistic range. 


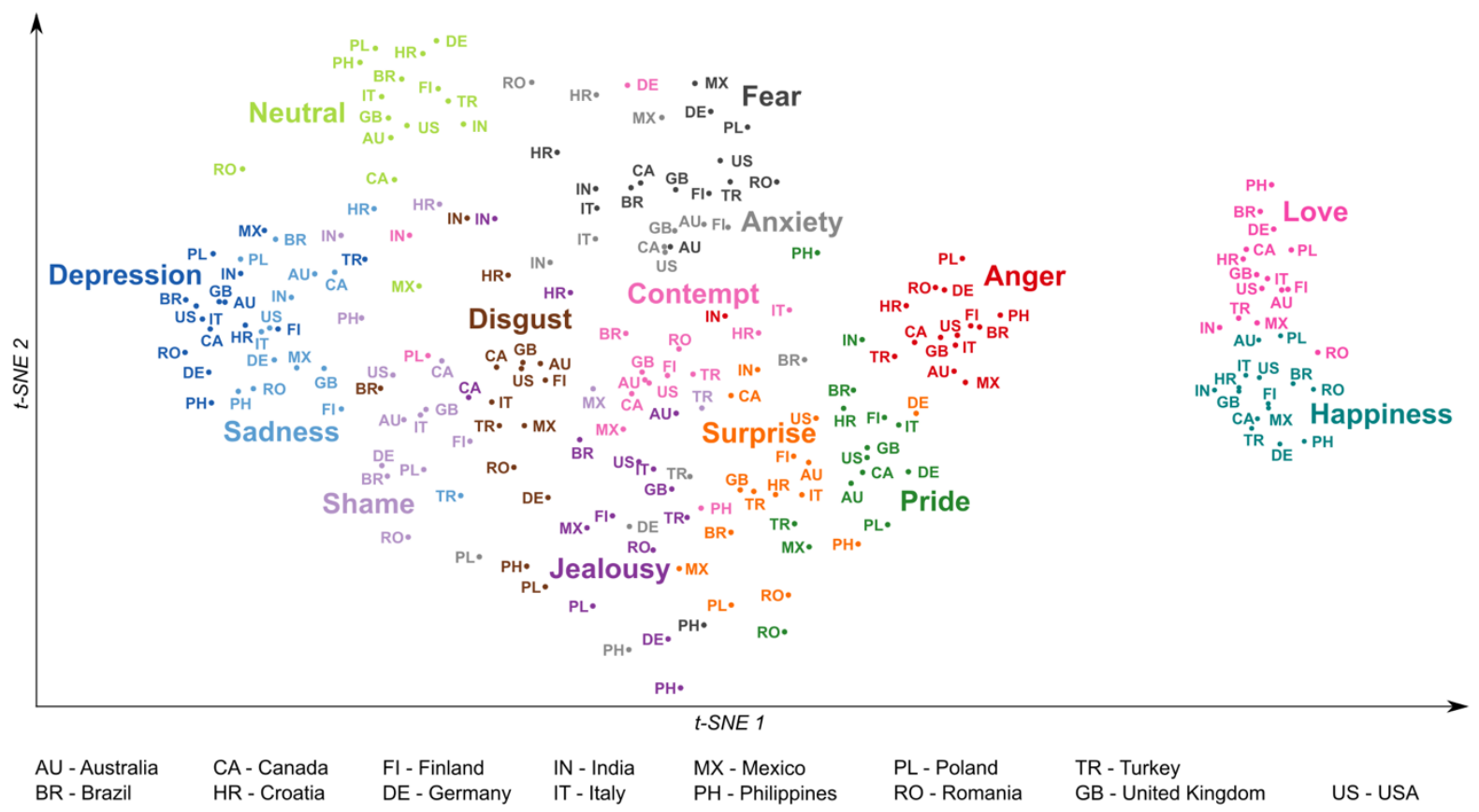

Figure 2. The t-SNE based multidimensional scaling projection of the BSM data for each

emotion in 15 countries with $\mathbf{N}>45$. Body maps which are more similar are closer in the t-SNE space.

Color coding indicates the a priori emotional categories denoted with larger words inside the clusters.

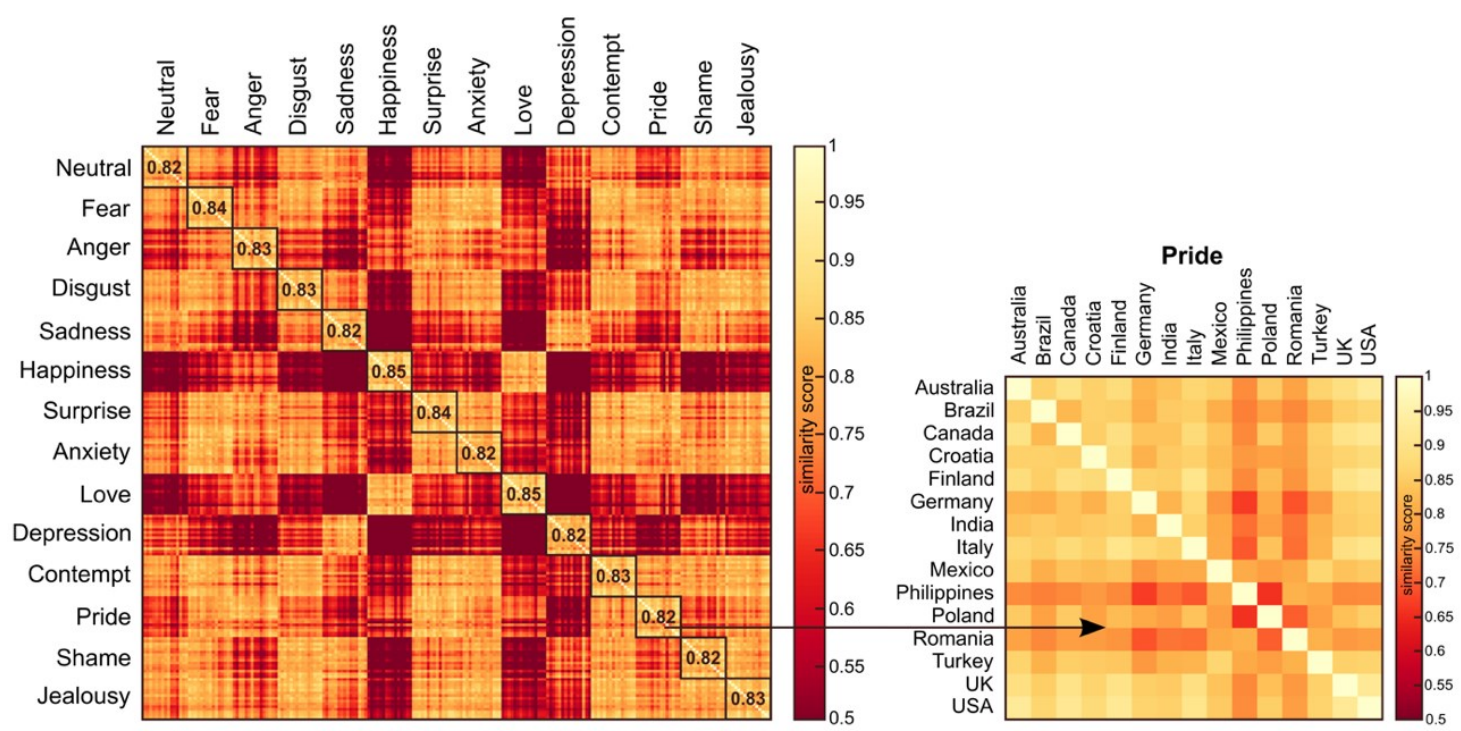

Figure 3. Similarity of effect-size bodily maps for each emotion + country pair (across 15

countries with $\mathbf{N}>45)$. Average similarity across countries per emotion is marked in the larger diagonal squares (ps < 0.001). The colorscale shows similarity values (Spearman correlations). The matrix on the right illustrates the order of the countries in the full similarity matrix. 
Ridge regression analysis confirmed that emotions themselves were the major source of variation in the BSMs, with factors relating to sociodemographics, anthropometrics, and culture contributing to the variance only modestly (Fig. S2). The only major exception was age: the BSMs showed an age-dependent decline in intensity (Fig. 4). Age correlated negatively with pixel-wise values of BSMs for anger, fear, disgust, happiness, surprise, anxiety, love, contempt, pride, shame and jealousy, and positively with BSMs for depression and sadness (for all BSMs, $r>0.036, p<0.05$, FDR corrected); because depression and sadness were associated with sensations of deactivation (negative values), the positive correlation thus implies dampening of the sensations of decreasing activity for these emotions. Accordingly, subjectwise mean absolute intensity scores (across all the pixels and emotions) were negatively associated with age $\left(r_{s}=0.11, p<0.001\right)$. No significant correlations were found between the subjects' BSMs and their geographical location (longitude and absolute latitude) and body parameters (height, weight, BMI). Two-sample t-test comparison however revealed some differences between BSMs of Western and Non-Western groups $(t>2.65$, $p<0.05$, FDR corrected; Fig. S3). Western subjects reported more activation in various regions of their bodies when feeling fear, anxiety, disgust, happiness, love, pride, contempt, jealousy, anger, as well as while being neutral. On the contrary, Non-Western subjects reported less deactivation for depression, sadness and shame. Differences between male and female subjects were modest $(t>2.65, p<0.05$, FDR corrected; Fig. S4). Compared with males, females reported more activation in their "guts" during anger, jealousy, anxiety and shame, and in their throat during anxiety, shame, fear, contempt, and sadness (more activation in the heart area as well). Females also reported less activation in their legs when feeling surprised, depressed and neutral. Males reported stronger activation in their genital area when experiencing love. 


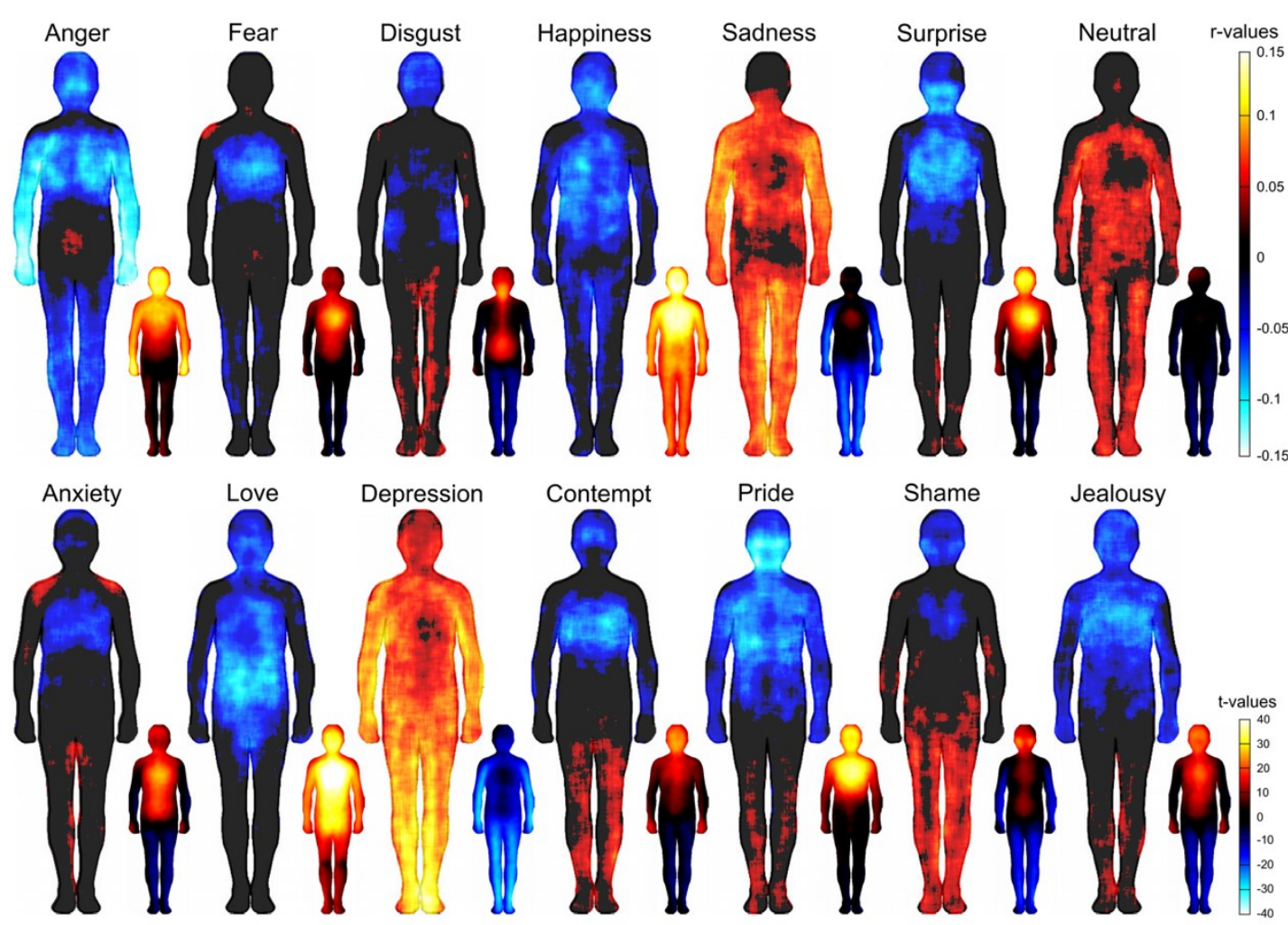

Figure 4. Age-dependent changes in bodily sensations for basic (upper row) and nonbasic

(lower row) emotions. On the large bodies, hot colours indicate positive and cool colours indicate negative correlations with age $(|\mathrm{r}|>0.036, \mathrm{p}<0.05$, FDR corrected). The small bodies show the mean bodily sensation maps for each emotion in the whole sample (from Fig.2) for the sake of reference. Note that age-dependent dampening is seen for all emotions: the emotion-related deactivations (e.g. for sadness and depression) have negative values in the mean body maps, thus their positive correlation with age reflects dampening of these sensations The upper colourbar indicates the r-statistic range for large bodies, the lower colourbar indicates the t-statistic range for small bodies

\section{Discussion}

Our main finding was that bodily sensations associated with different emotions are strikingly concordant across a wide range of cultures spanning the northern (e.g. Finland, Poland, Romania, Canada) and southern (Brazil, Mexico, Indonesia, Australia) hemispheres, as well as Western (e.g. USA, Canada), Latin American (e.g. Brazil, Mexico), Hindu (e.g. Indian), Orthodox (Romania), and Islamic (e.g. Turkey) cultures per the standard definition by Huntington 
(Huntington, 1996). Remarkably, the bodily sensation maps were also invariant across sexes and various sociodemographic factors. Altogether these results provide support for the view that subjectively felt emotions strongly reflect interoceptive processes (Damasio et al., 2000; Nummenmaa et al., 2014), and that emotions are experienced in the body in predominantly cultureindependent, discrete fashion, lending support to the basic emotion view (Ekman, 1992). Despite cultural and sex invariance, we however found significant age-dependent reduction in the intensities of the bodily felt emotions, reflected in decreasing sensations of both bodily activation and deactivation.

Across cultures, each emotion was associated with a unique and discernible "bodily fingerprint" (Fig. 2), in agreement with those reported in previous studies (Nummenmaa et al., 2014). Despite the minor differences in BSMs across countries (Fig. 3), the examined emotions form distinctive clusters with BSMs from different countries clustering together in the a priori emotion categories. This is further supported by the correlation-based similarity analysis (Fig. 4), highlighting clear concordance $(r s>0.82)$ for BSMs of each emotion across countries. The ridge regression analysis confirmed that the emotions themselves explained most variation in the BSMs, with cultural and anthropometric variables contributing only weakly (Fig. S2). These data accord with the view that emotions have a strong biological basis and they are only modestly influenced by cultural factors (Ekman, 1992; Sauter et al., 2010). This suggests that on subjectively experienced level, the universalism extends beyond the canonical "Ekman's 6 basic" emotions to a variety of non-basic or social emotions known to have discrete neural andexpressive bases (Cowen \& Keltner, 2017; Saarimaki et al., 2018; Shaw et al., 2005).

Previous research has found that cultural factors influence how people express and perceive emotions particularly on the face, questioning the universality of human emotions (Jack et al., 2012; Nelson \& Russell, 2013), However, recent investigations suggest that cultural similarity of a broad array (22) of facial expressions is more similar than different across cultures (Cordaro et al., 2018), and that even building blocks of socially complex emotions such as pride are culturally universal 
(Sznycer et al., 2017). Our results reconcile these diverging lines of evidence: while the nonverbal expressions of emotions may be malleable to cultural influences and learning-dependent plasticity, the embodied experience of emotions is universal (although see Russell, 1991). Because facial and vocal expressions, as well as gestures, can be seen and heard by other individuals, it is understandable that they may be more strongly influenced by sociocultural experiences than the bodily states associated with emotions. The latter — such as heart and breathing rate, muscle tension, and so forth (Kreibig, 2010) — go most of the time unnoticed by observers and are thus less likely to be transmitted from one individual to another. This dovetails nicely with recent findings suggesting that affective communication via body - particularly via social touching - is culturally universal (Suvilehto et al., 2015; Suvilehto et al., in press).

We found no clear sex differences in the bodily felt emotion, even though it has been previously suggested that males would be more internally focused with respect to their emotions. (Pennebaker \& Roberts, 1992). This overall sex-invariance however seems to contrast prior work showing sex differences in nonverbal emotional expressions and in the processing of emotional signals (Kret \& De Gelder, 2012). Yet, sex differences in subjectively felt emotions, as well as in autonomic nervous system responses during emotions, are less well established. Again, our results suggest that the expressive dimension may show more experience-dependent plasticity than the bodily felt emotions. This suggests that both sexes feel emotions in their bodies similarly, even though they might express those emotions differently. A recent review on emotional expressions in childhood and adolescence shows that the sex differences in expression of various emotions only begin to emerge through school-age and adolescence (Chaplin \& Aldao, 2013). These differences are influenced by contextual factors; differences between boys and girls are almost absent when the children interact with parents, but become significant during interaction with unfamiliar adults or peers (Chaplin \& Aldao, 2013). Thus, the sex differences in emotional expressions observed in adulthood may arise more from social learning and internalizing the sexappropriate emotional display rules, rather than from biological factors affecting the bodily feelings. 
If that were true, the physiology of emotions and related bodily sensations should indeed remain alike in males and females - as was observed in the present study - even when their expression patterns diverge.

Bodily sensations waned with ageing for all emotions. Alongside with our previous developmental work, showing that bodily feelings of emotions becoming more and more discrete from childhood toward early adulthood (Hietanen et al., 2015), these data reveal a dynamic developmental time course of emotional bodily feelings: After becoming discrete towards adulthood, these sensations slowly weaken towards the old age. Our data cannot provide a direct causal explanation for this effect, but multiple feasible possibilities exist. Basal metabolism and various physiological processes slow down during ageing (Schofield, 1985) and increased age is also coupled with decreased reactivity of the limbic system, increased activity of prefrontal cortex, slower cardiovascular response, and prolonged neuroendocrine response. These age-dependent physiological changes could explain our finding of the dampened bodily sensations of emotions during aging, and altogether these data could explain why subjective and physiological responses to emotional stimuli become decoupled during ageing (Scheibe \& Carstensen, 2010). Furthermore, our data could also explain why older people are in general better in emotion regulation (Urry \& Gross, 2010): due to weakened interoceptive awareness, they may become less connected to the bodily components of emotions, and this shift of emotions towards "cognitive" as opposed to embodied processes might make the emotions easier to regulate. Altogether these alterations might explain why ageing is generally associated with increased life satisfaction and emotional well-being.

\section{How universal are the bodily maps of emotions?}

Focus on null hypothesis testing is an inherent problem in cross-cultural research. As long as scientists are looking for differences between cultures, the null hypothesis easily becomes "no differences between cultures" which is impossible to prove using Fisherian statistics. This is particularly problematic, as cross-cultural research requires large samples, where trivially small 
differences may become statistically significant, even though the corresponding variables would not meaningfully increase the predictive accuracy of the model. This may bias scientists towards seeing small differences across cultures even when consistencies would dominate (see e.g. Hanel et al., 2019). In cross-cultural studies it would thus be better to focus on i) quantifying the de facto degree of similarity across cultures and ii) testing whether there is evidence for cross-cultural consistency based on some pre-defined criterion favouring universality, rather than testing if there is just any potentially trivial cultural variation. Unfortunately, there is no clear definition of how large "meaningful" cultural similarity in psychological studies should be. From practical viewpoint we can contemplate the presently observed effect size (ES) for cross-cultural consistency $(r=0.82)$ against ES with practical significance. For example, mean typical ES for common medications considered effective in the general population (levodopa treatment for Parkinson's disease, antipsychotics for schizophrenia, or antidepressants for major depressive disorder and so forth) are typically well below $r=0.40$ (Leucht et al., 2015). This is less than half than that of the ES for the consistency of bodily maps across cultures. Against such baseline, we would argue that the present data clearly favour universalism at least in the tested populations.

A second common problem in cross-cultural work pertains to the sampling of the highdimensional cultural space separated by national and geographic boundaries. We note that the present data did not cover all the world's cultures (defined as nations). The sample lacked sufficient number of, for example, East Asian and African respondents, which limits the generalizability of the findings to these populations. These cultures need to be sampled in detail in future studies. Although we have previously demonstrated that West European and East Asian bodily sensation maps are concordant (Nummenmaa et al., 2014), it is possible that in some (e.g. African) cultures tested in the future, the bodily maps would somehow differ from what was observed in the present sample. But how should such a potential discrepancy be interpreted if it was observed? One approach would be to consider this as proof by contradiction and take it as evidence against the universality of the bodily maps. This might be applicable if "universality" would mean strictly that 
a phenomenon is observed in exactly the similar fashion in all individuals and all cultures. Yet, our view of the psychological universality refers to the degree of consistency of psychological phenomena across cultures, rather than naïve all-or-nothing conceptualization. Accordingly, the question is not whether the bodily maps of emotions are different in some culture, but instead how similar they are across the world's population. In line with this reasoning, the question of cultural universality should not be treated as an yes-or-no phenomenon, but a continuum of cultural influences on the human nature, ranging from universal homeostatic and defensive responses to flexible and malleable social processes. This obviously calls for Bayesian reasoning in this type of studies, where the accumulation of evidence favouring different cultural universals versus discrepancies could be quantified.

Our data were based on self-reports. Even though humans are capable of sensing changes in their bodily states, interoceptive accuracy is far from perfect (Critchley et al., 2004) and bodily changes are not strongly coupled with subjective feelings of emotions (Mauss et al., 2005; Nummenmaa \& Saarimäki, 2017). Thus, the present data do not reflect a detailed description of emotion-specific physiological changes but the net consciously felt changes occurring in the body during emotions, and the BMs are thus subjective feeling maps. The Internet-based data acquisition was relatively uncontrolled and might have led to response bias due to uneven access to Internet across the targeted populations, attrition, and selection bias (for example, the sample was biased towards women and highly educated subjects). However this approach has been used successfully previously in mapping various interoceptive and somatosensory processes (Suvilehto et al., 2015) and the presently observed mean BSMs accord well with previous reports (Hietanen et al., 2015; Nummenmaa et al., 2014). Countries of residence were assessed based on the IP address; however, sampling in a subset of the data showed that IP address and self-reported nationality match on average on $85 \%$ of the cases. The task was presented in English to all subjects, which could potentially bias the results towards English-speaking immigrants in each country. Yet, using single language in the task circumvented confounds arising from translation. All in all, these are unlikely 
severe confounds, given that the overall bodily topographies of emotions match closely with those reported earlier and that any effects of culture on the body maps were minimal to begin with.

\section{Conclusions}

Emotions are associated with unique and discernible "bodily fingerprints" which are to large extent culturally universal. The intensity of these fingerprints however diminished during ageing. In turn, sex, anthropometric and sociodemographic variables affected the BSMs only weakly. These data support the view that emotions have a strong biological basis and that they are only modestly influenced by cultural factors. The results also suggest that, at bodily level, the universalism extends beyond the canonical "basic" emotions. Even though we found minor differences between Western and Non-Western groups, as well as between males and females, these effects were small in comparison with the typical intercultural consistency of the BSMs. We conclude that while the nonverbal expressions of emotions may be malleable to cultural influences, the embodied experience of emotions is likely more universal, because it cannot be directly observed and copied from others and likely reflects underlying physiological changes and interoceptive awareness.

Acknowledgements. This research was supported by the Academy of Finland grants \#294897 and \#304385 to LN. The funders had no role in study design, data collection and analysis, decision to publish, or preparation of the manuscript.

\section{References}

Anderson, C. L., et al. (2018). Emotion in the Wilds of Nature: The Coherence and Contagion of Fear During Threatening Group-Based Outdoors Experiences. Emotion, 18, 355-368.

Chaplin, T. M., et al. (2013). Gender Differences in Emotion Expression in Children: A MetaAnalytic Review. Psychol Bull, 139, 735-765.

Cordaro, D. T., et al. (2018). Universals and Cultural Variations in 22 Emotional Expressions across Five Cultures. Emotion, 18, 75-93.

Cowen, A. S., et al. (2017). Self-Report Captures 27 Distinct Categories of Emotion Bridged by Continuous Gradients. Proceedings of the National Academy of Sciences. 
Critchley, H. D., et al. (2004). Neural Systems Supporting Interoceptive Awareness. Nature neuroscience, 7, 189-195.

Damasio, A. R., et al. (2000). Subcortical and Cortical Brain Activity During the Feeling of SelfGenerated Emotions. Nature neuroscience, 3, 1049-1056.

Ekman, P. (1992). An Argument for Basic Emotions. Cogn Emot, 6, 169-200.

Fischer, A. H., et al. (2004). Gender and Culture Differences in Emotion. Emotion, 4, 87-94.

Folster, M., et al. (2014). Facial Age Affects Emotional Expression Decoding. Frontiers in Psychology, $5,13$.

Hanel, P. H. P., et al. (2019). A New Way to Look at the Data: Similarities between Groups of People Are Large and Important. J Pers Soc Psychol, 116, 541-562.

Hietanen, J. K., et al. (2015). Bodily Maps of Emotions across Child Development. Developmental Science, $\mathrm{n} / \mathrm{a}-\mathrm{n} / \mathrm{a}$.

Huntington, S. P. (1996). The Clash of Civilizations and the Remaking of World Order New Delhi: Penguin Books India.

Jack, R. E., et al. (2012). Facial Expressions of Emotion Are Not Culturally Universal. Proceedings of the National Academy of Sciences, 109, 7241-7244.

Kitayama, S., et al. (2006). Cultural Affordances and Emotional Experience: Socially Engaging and Disengaging Emotions in Japan and the United States. J Pers Soc Psychol, 91, 890-903.

Kragel, P. A., et al. (2016). Decoding Spontaneous Emotional States in the Human Brain. PLOS Biology, 14, e2000106.

Kreibig, S. D. (2010). Autonomic Nervous System Activity in Emotion: A Review. Biological Psychology, 84, 394-421.

Kret, M. E., et al. (2012). A Review on Sex Differences in Processing Emotional Signals. Neuropsychologia, 50, 1211-1221.

Leucht, S., et al. (2015). How Effective Are Common Medications: A Perspective Based on MetaAnalyses of Major Drugs. BMC Medicine, 13, 253.

Levenson, R. W. (2003). Blood, Sweat, and Fears - the Autonomic Architecture of Emotion. In P. Ekman, J. J. Campos, R. J. Davidson, \& F. B. M. DeWaal (Eds.), Emotions inside Out: 130 Years after Darwin's the Expression of the Emotions in Man and Animals (Vol. 1000, pp. 348-366). New York: New York Acad Sciences.

Manninen, S., et al. (2017). Social Laughter Triggers Endogenous Opioid Release in Humans. The Journal of Neuroscience, 37, 6125-6131.

Matsumoto, D., et al. (2008). Mapping Expressive Differences around the World - the Relationship between Emotional Display Rules and Individualism Versus Collectivism. J Cross-Cult Psychol, 39, 55-74.

Mauss, I. B., et al. (2005). The Tie That Binds? Coherence among Emotion Experience, Behavior, and Physiology. Emotion, 5, 175-190.

Mill, A., et al. (2009). Age-Related Differences in Emotion Recognition Ability: A Cross-Sectional Study. Emotion, 9, 619-630.

Nelson, N. L., et al. (2013). Universality Revisited. Emot Rev, 5, 8-15.

Norenzayan, A., et al. (2005). Psychological Universals: What Are They and How Can We Know? Psychol Bull, 131, 763-784.

Nummenmaa, L., et al. (2014). Bodily Maps of Emotions. Proc Natl Acad Sci U S A, 111, 646-651.

Nummenmaa, L., et al. (2018). Maps of Subjective Feelings. Proc Natl Acad Sci U S A, 115, 9198 9203.

Nummenmaa, L., et al. (2017). Emotions as Discrete Patterns of Systemic Activity. Neuroscience Letters.

Nummenmaa, L., et al. (2016). Topography of Human Erogenous Zones. Arch Sex Behav.

Pennebaker, J. W., et al. (1992). Toward a His and Hers Theory of Emotion - Gender Differences in Visceral Perception. Journal of Social and Clinical Psychology, 11, 199-212.

Russell, J. A. (1991). Culture and the Categorization of Emotions. Psychol Bull, 110, 426-450. 
Saarimaki, H., et al. (2018). Distributed Affective Space Represents Multiple Emotion Categories across the Human Brain. Soc Cogn Affect Neurosci.

Saarimäki, H., et al. (2018). Distributed Affective Space Represents Multiple Emotion Categories across the Human Brain. Soc Cogn Affect Neurosci, nsy018-nsy018.

Saarimäki, H., et al. (2016). Discrete Neural Signatures of Basic Emotions. Cereb Cortex, 6, 25632573.

Sauter, D. A., et al. (2010). Cross-Cultural Recognition of Basic Emotions through Nonverbal Emotional Vocalizations. Proc Natl Acad Sci U S A, 107, 2408-2412.

Scheibe, S., et al. (2010). Emotional Aging: Recent Findings and Future Trends. J Gerontol Ser BPsychol Sci Soc Sci, 65, 135-144.

Schofield, W. N. (1985). Predicting Basal Metabolic Rate, New Standards and Review of Previous Work. Hum Nutr Clin Nutr, 39 Suppl 1, 5-41.

Scott, S. K., et al. (2015). The Social Life of Laughter. Trends Cogn Sci, 18, 618-620.

Shaw, P., et al. (2005). Differential Effects of Lesions of the Amygdala and Prefrontal Cortex on Recognizing Facial Expressions of Complex Emotions. J Cogn Neurosci, 17, 1410-1419.

Siegel, E. H., et al. (2018). Emotion Fingerprints or Emotion Populations? A Meta-Analytic Investigation of Autonomic Features of Emotion Categories. Psychol Bull, 144, 343-393.

Suvilehto, J., et al. (2015). Topography of Social Touching Depends on Emotional Bonds between Humans. Proc Natl Acad Sci U S A, 112, 13811-13816.

Suvilehto, J., et al. (in press). Cross-Cultural Similarity in Relationship-Specific Social Touching. Proceedings of the Royal Society Series B-Biological Sciences.

Sznycer, D., et al. (2017). Cross-Cultural Regularities in the Cognitive Architecture of Pride. Proc Natl Acad Sci U S A, 114, 1874-1879.

Thompson, R. A., et al. (2006). Feeling and Understanding: Early Emotional Development. In K. McCartney \& D. Phillips (Eds.), The Blackwell Handbook of Early Childhood Development (pp. 317-337). Oxfrod: Blackwell.

Torregrossa, L. J., et al. (2018). Anomalous Bodily Maps of Emotions in Schizophrenia.

Urry, H. L., et al. (2010). Emotion Regulation in Older Age. Current Directions in Psychological Science, 19, 352-357.

Widen, S. C. (2013). Children's Interpretation of Facial Expressions: The Long Path from ValenceBased to Specific Discrete Categories. Emot Rev, 5, 72-77. 